\title{
Prestação de Serviços de Manutenção Predial em Estabelecimentos Assistenciais de Saúde
}

\author{
Provision of building maintenance services in healthcare facilities
}

Gláucia M aria Amorim ${ }^{1}$

Eliana Cardoso V ieira Quintão ${ }^{2}$

Herćlio M artelli Júnior ${ }^{3}$

Paulo Rogério Ferreti Bonan ${ }^{3}$

${ }^{1}$ Fundação H ospitalar do Estado de M inas Gerais. Alameda Álvaro Celso 100 Santa Efigênia. 30150-260 Belo Horizonte M G.

glauciamamorim@gmail.com

${ }^{2}$ Empresa DNV

${ }^{3}$ Departamento de

Odontologia, Universidade

Estadual de M ontes Claros.
Abstract The scope of this paper was to evaluate the provision of building maintenance services in health units, by means of a descriptive, quantitative and cross-sectional study, considering the five types of facilities (Primary H ealth, Emergency, Specialty, H ospital and M ental H ealth U nits). The research was approved by the Research Ethics Comittee of FHEM IG with the Terms of Agreement signed with the U nified Health System of Betim. Comparative analysis was conducted by checking the requirements of "Physical-Functional Structure M anagement" of the "Brazilian Hospital Accreditation M anual" of the $\mathrm{N}$ ational Accreditation Organization. Nonconformities were noted in the physical-functional manage ment of the health centers, especially the primary health units. The assessment was important, considering that compliance with formal, technical and structural requirements, welfare activities, according to the service organization and appropriate to the profile and complexity, can collaborate to minimize the risks of users. To improve the quality of health care establishments, it is essential that managers, backed by "top management," prioritize financial, human and material resources in planning to ensure compliance with security requirements of users in buildings. Key words Building maintenance, Provision of services, $M$ aintenance management, Physical planning, $\mathrm{H}$ ealth services
Resumo 0 objetivo do trabalho foi avaliar (através de estudo descritivo, quantitativo e transversal) a prestação deserviços de manutenção predial em U nidades de Saúde, considerando os cinco tipos de Estabel ecimentos (U nidade Básica de Saúde, U nidade de Pronto Atendimento, Especialidade, Hospitalar e Saúde M ental). A pesquisa foi aprovada no CEP da FHEMIG com o Termo de Concordância junto ao SUS Betim. Foi realizada a análise comparativa através da verificação de requisitos da "Gestão da Estrutura Físico-Funcional", do "M anual Brasileiro de Acreditação Hos pitalar" da ON A. Foram constatadas inconformidades na gestão físico-funcional dosEAS, especialmente das U BS. I mportante essa avaliação, considerando que o cumprimento dos requisitos formais, técnicos e de estrutura, as atividades assistenciais, de acordo com a organização do serviço e adequadas ao perfil eà complexidade, podem colaborar para minimizar os riscos dos usuários. Para a melhoria da qualidade assistencial dos estabelecimentos, é imprescindível que os gestores, com 0 respaldo da "alta direção", priorizem, nos planejamentos, os recursos financeiros, humanos e materiais a fim de garantir o cumprimento das exigências da segurança dos usuários nos edifícios. Palavras-chave M anutenção predial, Prestação de serviços, Gestão da manutenção, Planejamento físico, Serviços de saúde 
Introdução

A análise de um sistema de saúde deve focar a estruturação e o funcionamento, as articulações institucionais que apresenta, de acordo com as qualificações em relação à população abrangida e ao modelo assistencial implementado, assim como as formas de financiamento adotadas ${ }^{1}$. Anteriormente, a redede saúdeno Brasil eracentrada na produção de procedimentos médicos e diagnósticos, desintegrada, distribuída de forma inadequada segundo as regiões com as suas diversas características. 0 recente processo de municipalização da saúde brasileira vem viabilizando condições de melhoria, como resultado da participação das comunidades e dos movimentos sociais, possibilitando, assim, que a atenção primária seja priorizada, inclusive com a criação de normas e decretos relativos a novas formas de financiamentos públicos, e viabilizando aos gestores da saúde o cumprimento do preceito constitucional da integralidade 2 . Nos sistemas fragmentados de atenção à saúde vigora a visão de uma estrutura hierárquica definida por níveis de "complexidades" crescentes e com relações de ordem e graus de importância entre os diferentes níveis do SUS, segundo suas normativas em atenção básica, aten ção de média e de alta complexidade. Essa visão é equivocada ao estabelecer que a atenção primária à saúde seja menos complexa do que a atenção nos níveis secundário e terciário ${ }^{3}$. Existe, portanto, a mudança de paradigma, que é o conceito de "Rede de Atenção à Saúde" (RAS), com a decisão norteada por evidências dos sistemas fragmentados, voltados para as condições e os eventos agudos, para as redes de saúde, com foco nas condições agudas e crônicas. Experiências bem-sucedidas em outros países da União Europeia, nos Estados Unidos e no Canadá devem ser adequadas à realidade do sistema público brasileiro. 0 incremento dos re cursos financeiros para o SUS deve vir junto com mudanças que possam alinhar os incentivos econômicos com os objetivos sanitários. 0 sistema de pagamento por procedimentos sinaliza para os prestadores de serviços que ofertem mais serviços e mais caros para maximizar seus ganhos, o que os estimula a oferecer, não o cuidado ne cessário, mas o máximo de cuidado possível, ao tempo em que os desestimulam a ofertar intervenções voltadas para a promoção e a manutenção da saúde, fortalecendo, assim, um sistema de doença em detrimento de um sistema de saúde. Esse desalinhamento entre os objetivos do sistema de atenção à saúde e os seus mecanismos de financiamento explica o fato de que diversos procedimentos de saúde ofertados são desnecessários do ponto de vista sanitário ${ }^{3}$. 0 financiamento das redes envolve: 0 custeio com foco em mecanismos de pagamento para cobrir as necessidades e as demandas de saúde da sua população; 0 investimento na compatibilização da capacidade física com as necessidades da população e a racionalização da estrutura operacional de acordo com a capacidade fiscal e financeira e com o planejamento estratégico de investimentos para a rede. 0 financiamento dos componentes das RAS engl oba o custeio eo alinhamento dos incentivos econômicos para favorecer a responsabilização compartilhada entre todos esses componentes; já o investimento, o direcionamento para os serviços de saúde, é feito segundo o plano estratégico, contemplando os princípios deeconomia deescalae deescopo em consonância com a qualidade 3 .

0 aspecto físico dos Estabelecimentos Assistenciais deSaúde(EAS) estáintimamentevinculado à funcionalidade. Os processos inerentes à arquitetura, à engenharia, à organização, à administração, à manutenção, à bioengenharia, à enge nharia clínica, à informática, à organização, à administração, focados na área hospitalar, são meios, procedimentoserecursosque, juntamentecom os profissionais de saúde, têm como finalidade viabilizar o objetivo comum para a proteção, a promoção e a recuperação da saúde dos usuários dos edifícios construídos para o fim assistencial4. 0 ambientefísico tem impacto significativo sobre a saúde e a segurança, no entanto, os hospitais não foram concebidos com o objetivo explícito de aumentar a segurança do paciente por meio de projeto de instal ações 5 . Daí a importância de avaliar os aspectos físico-funcionais dos estabelecimentos construídos para fins assistenciais.

Avedis Donabedian, na década de 60, definiu que a garantia da qualidade em saúde significa o esforço permanente realizado no melhoramento da saúde, pela monitorização e avaliação continuada da estrutura, do processo e dos resultados da prestação dos serviços. Baseada nesse conceito, a avaliação, segundo o manual da ONA, considera que a estrutura, os processos e os re sultados devem ser garantidos nos três níveis, cujos princípios são segurança, sistema de gestão integrado e excelência ${ }^{6} 0$ manual écomposto por seções que se desdobram em subseções e cada uma tem um padrão definido que deve ser evidenciado no processo de avaliação. 0 nível 2 traz o conceito de efetividade: manter-se sustentável no ambiente, apresentar resultados globais 
ao longo do tempo, cumprir o dever eaumentar o lucro (eficiência mais eficácia). Assim, as avaliações têm foco na busca da evidência de que os processos são eficazes, ou seja, conseguem atingir os seus objetivos, deforma eficiente, utilizando adequadamente os recursos, com o objetivo de atingirem melhores resultados ao longo do tempo. Resultado corresponde às consequências das atividades do serviço de saúde ou dos profissionais, em termos da melhoria do nível de saúde, da capacidade funcional restaurada, do alívio do sofrimento e da satisfação do cliente ${ }^{6}$.

Considerando que as pesquisas relativas à manutenção predial em EAS são escassas, além das deficiências constatadas na revisão literária, foi oportuna a escolha do tema. Outra situação prática favorável ao presente estudo foram as atividades da pesquisadora como arquiteta junto ao SUS Betim e à FHEM IG, o que determinou a escolha dos Estabelecimentos pesquisados. Outro fator facilitador foi a colaboração de uma profissional auditora para a capacitação da pesquisadora na verificação dos quesitos da "gestão físico-funcional" em um Estabelecimento deSaúde, sendo esta, por conseguinte, a coleta amostral.

Com o propósito de realizar um levantamento situacional, este estudo objetivou avaliar, de forma comparativa, a prestação de serviços de manutenção predial em EAS localizados no Estado de M inas Gerais, uma vez que esse processo éimprescindível à garantia da segurança dosusuários dos edifícios assistenciais.

\section{M etodologia}

O objetivo do trabalho foi avaliar, por meio de estudo descritivo, quantitativo e transversal, a prestação deserviços de manutenção predial em Unidades de Saúde, considerando os cinco tipos de Estabelecimentos (Unidade Básica de Saúde, Unidade de Pronto Atendimento, Especialidade, H ospitalar e Saúde M ental). Realizado no período de julho a novembro de 2011, em 61 EAS localizados no Estado de M inas Gerais, Brasil (Tabela 1), o estudo foi feito por meio da verificação de quesitos da "Gestão Físico-Funcional”, já validada, adotados no "M anual Brasileiro de Acreditação H ospitalar da Organização Nacional de Acreditação", ONA-3, versão 20106,7. Nos roteiros para a acreditação dos Estabel ecimentos, são adotadas várias verificações em diversos quesitos; entretanto, no estudo em questão, foram utilizados aqueles referentes aos processos inerentes à infraestrutura. Para o cálculo de associ- ação entre as variáveis coletadas, foi utilizado 0 "teste Qui-quadrado" quando apropriado, e o "teste exato de Fisher", quando necessário. Tais testes indicam se há ou não associação significativa entre variáveis categóricas.

Constituem os 61 EAS pesquisados:

- Clínica de Hematologia (Especialidade), coleta amostral mediante a orientação da médica Eliana Quintão de como se faz uma auditoria baseada em requisitos do "M anual Brasileiro de Acreditação para Organizações Prestadoras de Serviço de Saúde", versão 2010, da ONA. A clínica foi acreditada pela ONA, "acreditação plena, nível 2", Empresa DNV/junho $2010^{6}$.

- 57 EAS da rede SUS Betim: 34 UBSs, 12 Especialidades, 5 Saúde M ental, 4 U PAs e 2 H ospitalares.

- 3 unidades hospitalares da FHEM IG (total 25), que se encontram em processo de acreditação pela ONA. O H ospital Regional Antônio Dias, localizado em Patos de Minas, recebeu o selo ONA de acreditação plena nível 1 - DNV, em 2011.

Os quesitos com os itens de verificação foram acompanhados pelos responsáveis dos serviços demanutenção predial medianteconsentimento formal e assinado em um Termo de Consentimento. Esta pesquisa foi aprovada pelo ComitêdeÉtica em Pesquisa (CEP) daFHEM IG. E, mediante Termo de Concordância assinado pela Secretaria M unicipal de Betim, foi autorizada também a pesquisa do SUS Betim.

\section{Resultados ediscussão}

Na Tabela 2 estão os dados referentes às características gerais das instituições, aos aspectos assistenciais efísicos que são pré requisitos para se candidatar ao processo de avaliação para Acreditação pela O NA. Foram verificados documen-

Tabela 1. Estabelecimentos de saúde agrupados analisados. M inas Gerais-2011.

\begin{tabular}{lrc}
\hline $\begin{array}{c}\text { Estabelecimentos } \\
\text { de Saúde }\end{array}$ & Frequência & Porcentagem \\
\hline UBS & 34 & 55,7 \\
Unidades especializadas & 13 & 21,3 \\
Unidades hospitalares & 5 & 8,2 \\
Unidades saúde mental & 5 & 8,2 \\
UPA & 4 & 6,6 \\
Total & 61 & 100,0 \\
\hline
\end{tabular}

Fonte: Autora. 
tos relativos aos projetos físicos nos órgãos ne cessários (regularização urbana municipal, VISA, Corpo de Bombeiros), assim como a composição e a organização da equipe responsável pela gestão da manutenção predial do edifício.

Observa-se que nem todos os EAS atendem aos pré requisitos exigidos para 0 atendimento do princípio da segurança dos usuários do Edifício, conforme o nível 1 do M anual de Acreditação da ONA, quais sejam:

- Responsável técnico registrado (50\%) eexistência de alvarás de funcionamento (49\%) e sanitário (48\%), quesitos, em geral, não atendidos pelas UBS e U PA. Segundo a RDC 63, de 25/11/ $2011^{8}$, os estabelecimentos integrantes da Administração Pública ou por ela instituídos independem da licença para funcionamento, ficando sujeitos, porém, às exigências pertinentes às instalações, aos equi pamentos eà aparelhagem adequada eà assistência e responsabilidade técnicas, aferidas por meio de fiscalização realizada pelo órgão sanitário local. Entretanto, a ONA define como uma das diretrizes, para a contratação do processo de avaliação de diagnóstico e de certificação, possuir alvará de funcionamento, licença sanitária e registro do responsável técnico, independenteda esfera administrativa en quadrada. Na prática, foi constatado nos hospitais e unidades especializadas (Farmácia, Banco de Leite, hematologia) que os profissionais médicos e farmacêuticos estão atentos à exigência de seu registro como responsáveis técnicos perante seus consel hos de classe.

- Todos os EAS possuem Cadastro Nacional de Estabelecimento de Saúde (CNES) ativo. Este é a base para operacionalizar os sistemas de informaçõ̃es em saúde para o gerenciamento eficaz e eficiente. Propicia ao gestor o conhecimento da realidade da rede assistencial esuas potencialidades, visando auxiliar no planejamento em saúde, dar visibilidade ao controle social a ser exercido pela população, além de disponibilizar informações das condições de infraestrutura de funcionamento dos EAS. Entretanto, em alguns cadastros consultados pela pesquisadora na internet, foram constatadas deficiências de dadose informações desatualizadas.

- "Projeto de Prevenção e Combate a Incêndio e Pânico": 21,3\% estão aprovados pelo Corpo de Bombeiros, um quesito fundamental para a segurança dos usuários nos edifícios. Na Tabe la 3 constata-se que $36,1 \%$ dos EAS possuem "sinalização defácil entendimento evisualização que oriente 0 acesso a todas as áreas da organização, com controle nas áreas restritas"; $24,6 \%$ não possuem. 57,4\% atendem ao quesito "condições de segurança e visualização clara e de fácil compreensão para obras, atividades de conservação, manutenção esituações deemergência". Essesitens

Tabela 2. Características gerais das instituições, aspectos assistenciais e físicos ( $n=61)$. M inas Gerais, 2011.

\begin{tabular}{|c|c|c|c|c|c|}
\hline Variáveis & $\underset{(n / \%)}{\operatorname{Sim}}$ & $\begin{array}{l}\text { Não } \\
(\mathrm{n} / \%)\end{array}$ & $\begin{array}{l}\text { Parcial } \\
(\mathrm{n} / \%)\end{array}$ & $\begin{array}{l}\text { Não se } \\
\text { aplica } \\
(\mathrm{n} / \%)\end{array}$ & $\begin{array}{l}N a ̃ o \\
\text { informado } \\
(\mathrm{n} / \%)\end{array}$ \\
\hline Tem responsável técnico registrado? & $11 / 18$ & $50 / 82$ & $0 / 0$ & 0 & 0 \\
\hline Possui Cadastro Nacional de Estabelecimento de Saúde (CNES) *ativo? & $61 / 100$ & $0 / 0$ & $0 / 0$ & 0 & 0 \\
\hline Possui Alvará de Funcionamento? & $9 / 14,8$ & $49 / 80,3$ & $0 / 0$ & 0 & $3 / 4,9$ \\
\hline Possui Alvará Sanitário? & $9 / 14,8$ & $48 / 78,6$ & $2 / 3,3$ & 0 & $2 / 3,3$ \\
\hline Possui projeto arquitetônico aprovado na Vigilância Sanitária? & $13 / 21,3$ & $31 / 50,8$ & $1 / 1,6$ & 0 & $16 / 26,74$ \\
\hline $\begin{array}{l}\text { Possui projeto arquitetônico aprovado na Prefeitura de acordo com a } \\
\text { regulação urbana? }\end{array}$ & $2 / 3,3$ & $59 / 96,7$ & $0 / 0$ & 0 & 0 \\
\hline $\begin{array}{l}\text { Projeto de Prevenção de Combate a Incêndio e Pânico aprovado pelo } \\
\text { Corpo de Bombeiros? }\end{array}$ & $13 / 21,3$ & $31 / 50,8$ & $6 / 9,8$ & 0 & $8 / 18,1$ \\
\hline Projeto elétrico aprovado na CEMIG? & $61 / 100$ & $0 / 0$ & $0 / 0$ & 0 & 0 \\
\hline Responsável pela equipe de manutenção predial? & $61 / 100$ & $0 / 0$ & $0 / 0$ & 0 & 0 \\
\hline Equipe de manutenção é a mesma de equipamentos? & $57 / 93,4$ & $4 / 6,6$ & $0 / 0$ & 0 & 0 \\
\hline Existem serviços terceirizados para a manutenção predial? & $56 / 91,8$ & $5 / 8,2$ & $0 / 0$ & 0 & 0 \\
\hline $\begin{array}{l}\text { Programa de Prevenção de Riscos Ambientais (PPRA) atualizado e } \\
\text { implantado? }\end{array}$ & $6 / 9,8$ & $53 / 86,9$ & $2 / 3,3$ & 0 & 0 \\
\hline $\begin{array}{l}\text { Plano de Gerenciamento de Resíduos de Serviços de Saúde implantado } \\
\text { (PGRSS)? }\end{array}$ & $8 / 13,1$ & $32 / 52,5$ & $19 / 31,1$ & 0 & $2 / 3,3$ \\
\hline
\end{tabular}

Fonte: Autora. 
Tabela 3. Requisitos da gestão da estrutura físico-funcional ( $n=61)$. M inas Gerais, 2011.

\begin{tabular}{|c|c|c|c|c|c|}
\hline Variável & $\begin{array}{l}\operatorname{Sim} \\
(\mathrm{n} / \%)\end{array}$ & $\begin{array}{l}\text { Não } \\
(\mathrm{n} / \%)\end{array}$ & $\begin{array}{l}\text { Parcial } \\
(\mathrm{n} / \%)\end{array}$ & $\begin{array}{l}\text { Não se } \\
\text { aplica } \\
(\mathrm{n} / \%)\end{array}$ & $\begin{array}{l}\text { Não } \\
\text { informado } \\
(n / \%)\end{array}$ \\
\hline $\begin{array}{l}\text { Profissionais com capacitação compatível, dimensionadas às } \\
\text { necessidades do serviço }\end{array}$ & $2 / 3,3$ & $2 / 3,3$ & $57 / 93,4$ & 0 & 0 \\
\hline $\begin{array}{l}\text { Condições operacionais e de infraestrutura para execução das } \\
\text { atividades, considerando o perfil e a complexidade da organização }\end{array}$ & $2 / 3,3$ & $0 / 0$ & $59 / 96,7$ & 0 & 0 \\
\hline $\begin{array}{l}\text { Gerencia as condições de infraestrutura físico-funcional para a } \\
\text { segurança dos profissionais, clientes e circundantes }\end{array}$ & $57 / 93,4$ & $4 / 6,6$ & $0 / 0$ & 0 & 0 \\
\hline Planejamento de obras & $15 / 24,6$ & $1 / 1,6$ & $45 / 73,8$ & 0 & 0 \\
\hline Projetos atualizados e em conformidade com o edificado & $16 / 26,2$ & $27 / 44,3$ & $6 / 9,8$ & 0 & 12 \\
\hline Condições de acessibilidade para pessoas com necessidades especiais & $22 / 36,1$ & $2 / 3,3$ & $37 / 60,7$ & 0 & 0 \\
\hline $\begin{array}{l}\text { Condições de segurança e sinalização clara e de fácil compreensão } \\
\text { para obras, atividades de conservação, manutenção e situações de } \\
\text { emergência }\end{array}$ & $35 / 57,4$ & $2 / 3,3$ & $24 / 39,3$ & 0 & 0 \\
\hline $\begin{array}{l}\text { Sinalização de fácil entendimento e visualização que oriente o acesso } \\
\text { a todas as áreas da organização, com controle nas áreas restritas }\end{array}$ & $22 / 36,1$ & $15 / 24,6$ & $24 / 39,3$ & 0 & 0 \\
\hline Saídas de emergência claramente sinalizadas e de fácil compreensão & $21 / 34,4$ & $39 / 63,9$ & $1 / 1,6$ & 0 & 0 \\
\hline M onitora a limpeza e desinfecção dos reservatórios de água & $28 / 45,9$ & $14 / 23,0$ & $15 / 24,6$ & 0 & 4 \\
\hline $\begin{array}{l}\text { M onitora a análise e o controle da potabilidade da água } \\
\text { periodicamente }\end{array}$ & $10 / 16,4$ & $48 / 78,7$ & $2 / 3,3$ & 1 & 0 \\
\hline $\begin{array}{l}\text { Gerencia, opera e controla os sistemas de água, energia elétrica, gases, } \\
\text { vapores, efluentes líquidos, proteção contra descarga elétrica, } \\
\text { climatização, combate a incêndio, alerta e emergência, telefonia e } \\
\text { rede lógica }\end{array}$ & $16 / 26,2$ & $0 / 0$ & $45 / 73,8$ & 0 & 0 \\
\hline $\begin{array}{l}\text { Gerencia, opera e controla os equi pamentos de infraestrutura } \\
\text { (elevadores, caldeiras, geradores de energia, monta-cargas, entre } \\
\text { outros), de acordo com o porte e dimensionamento às necessidades } \\
\text { do serviço }\end{array}$ & $8 / 13,1$ & $1 / 1,6$ & $4 / 6,6$ & 48 & 0 \\
\hline $\begin{array}{l}\text { Sistemas de conservação e recuperação da estrutura física predial e do } \\
\text { mobiliário, de acordo com o porte e dimensionado às necessidades do } \\
\text { serviço }\end{array}$ & $42 / 68,9$ & $4 / 6,6$ & $15 / 24,6$ & 0 & 0 \\
\hline $\begin{array}{l}\text { Sistemática de manutenção preventiva e corretiva das instalações e } \\
\text { dos equipamentos }\end{array}$ & $9 / 14,8$ & $2 / 3,3$ & $50 / 82$ & 0 & 0 \\
\hline Sistemática de calibração dos equipamentos & $51 / 83,6$ & $1 / 1,6$ & $2 / 3,3$ & 3 & 4 \\
\hline $\begin{array}{l}\text { Gerência riscos* assistenciais, sanitários, ambientais, ocupacionais e } \\
\text { responsabilidade civil (**envolve os ciclos de prevenção, detecção e } \\
\text { mitigação do risco visando um sistema seguro) }\end{array}$ & $61 / 100$ & $0 / 0$ & $0 / 0$ & 0 & 0 \\
\hline Planos de contingências & $5 / 8,2$ & $1 / 1,6$ & $52 / 85,2$ & 0 & 3 \\
\hline Processo Acreditação da ONA - Organização Nacional de Acreditação & $5 / 8,2$ & $55 / 90,2$ & $1 / 1,6$ & 0 & 0 \\
\hline
\end{tabular}

Fonte: Autora.

são imprescindíveis à segurança dos usuários, que, já se encontrando em situação de "cuidados", não podem estar "vulneráveis a riscos". Atualmente, não se exige apenas a execução dos serviços incluídos no projeto aprovado, o EAS tem que ser vistoriado e ministrar treinamentos constantes, mediante a simulação de possíveis eventos, com o objetivo de reduzir os riscos de forma preventiva e para que usuários, especialmente os trabalhadores, no caso de terem que enfrentar a situação, estejam preparados para controlar as chamas do fogo com segurança, pois, na maioria das vezes, o pânico dos quetentam se salvar acarreta mais vítimas que o próprio acidente.

- 9,8\% dos EAS possuem PPRA atualizado e implantado. A N orma Regulamentadora 9 (NR 9), por meio da Consolidação das Leis do Trabalho (CLT), no título "Programa de Prevenção de Riscos Ambientais", estabelece a obrigatoriedade da elaboração e implementação de um programa de "Higiene $\mathrm{O}$ cupacional" visando à preservação da saúde e à integridade física dos trabalhadores pela antecipação, reconhecimento, 
avaliação e consequente controle da ocorrência de riscos ambientais existentes ou que venham a existir no ambiente de trabal ho.

-13,1\% possuem PGRSS elaborados e implantados, apesar das exigências legais (RDC 306/ 2004 da ANVISA ${ }^{9}$ e CONAM A 358/2005 ${ }^{10}$ ). Esse documento aponta e descreve as ações relativas ao manejo dos resíduos sólidos, observadas as suas características, contem plando os aspectos referentes à geração, à segregação, ao acondicionamento, à coleta interna, ao armazenamento, ao transporte, ao tratamento e à destinação final, bem como os aspectos relativos à proteção à saúde pública eà segurança ocupacional do pessoal envolvido nas etapas do gerenciamento de resíduos.

$\mathrm{Na}$ Tabela 3, as variáveis se referem aos re quisitos do padrão do nível 1 do Manual da ONA, requisitos formais, técnicos e de estrutura para as atividades, que asseguram uma estrutura físico-funcional adequada ao perfil e à complexidade da organização, minimizando riscos e promovendo a ambiência. $\mathrm{Na}$ tabela existe um item que identifica os EAS em Processo de Acreditação da ONA (5 dos 61 pesquisados). 0 Hospital Regional de Betim iniciou seu processo de acreditação para participar do "Prêmio Célio de Castro", que consiste no reconhecimento dos hospitais integrantes do PRO-H OSP destacados em relação à gestão da qualidade e que tenham apresentado as melhores práticas, com base em critérios definidos pela ONA epela ANVISA, processo ainda não finalizado.

Pelos resultados obtidos quanto à "Gestão da Estrutura Físico-Funcional", constata-se que, em diversos EAS, vários requisitos formais, técnicose deestrutura ainda não são atendidos, quais sejam:

- 3,3\% possuem "condições operacionais e de infraestrutura para execução das atividades, considerando-seo perfil ea complexidadeda organização", e 96,7\% atendem parcial mentea esse que sito, que é uma condição básica e situação imprescindível para a gestão físico-funcional.

- A maioria (93,4\%) gerencia as condições de infraestrutura física para a segurança dos profissionais, clientes e circundantes, considerando que a atividade assistencial preconiza ações de cuidados que, Ihes sendo inerentes, tornam os gestores vigilantes nesse aspecto.

- 24,6\% efetuam o planejamento das obras; a maioria $(73,8 \%)$ cumpre parcialmente o quesito.

- 26,2\% possuem projetos atualizados e em conformidade com o edificado, situação imprescindível para viabilizar e facilitar a gestão física do prédio, pois é necessário o cadastro de todas as instal ações físicas com vistas à identificação de problemas para as possíveis e necessárias manutenções preventivas e corretivas, além de obras de reforma e acréscimos.

- 36,1\% possuem condições de acessibilidade para pessoas portadoras de necessidades especiais. Antes das orientações legais da NBR 9050/ 2004 ${ }^{11}$, existiam as exigências técnicas da RDC 50 de 21/02/2002 mento Técnico para planejamento, programação, elaboração e avaliação de projetos físicos de estabelecimentos assistenciais de saúde" -, nas quais se estabeleciam os quesitos construtivos para permitir a acessibilidade a portadores de necessidades especiais. Item estetambém contemplado nas exigências das VISA para a aprovação de construções deestabelecimentos desaúde, uma vez que os usuários dos estabelecimentos podem ser portadores, temporariamente, de dificuldades de locomoção, de necessidades especiais, eas exigências construtivas são itens para permitir o acesso e o fluxo de forma universal.

- 13,1\% gerenciam, operam e controlam os equipamentos de infraestrutura; um estabelecimento deveria ter elevador para o fluxo de usuários entre dois pavimentos; 4 U PA não possuíam gerador, mas se encontravam em processo de aquisição por exigência legal, na época da pesquisa, para começar a receber recursos do $\mathrm{M} \mathrm{i-}$ nistério da Saúdepara custeio. Em outros 48EAS, a exigência não se aplica como em todas as unidades básicas e algumas de especialidades.

- 14,8\% apresentam a sistemática de manutenção preventiva e corretiva das instalações e dos equipamentos. Existe manutenção corretiva na maior parte destes, mas não existem equipes com profissionais em quantidadesuficienteecom capacitação adequada para a programação dos serviços preventivos, fundamentais para evitar transtornos que comprometem, inclusive, o funcionamento e 0 atendimento adequado nas unidades assistenciais. A "M anutenção Administrativa ou Programada", devidamente organizada, estrutura e assessora, inclusive com a colaboração de especialistas contratados que planejam, realizam eimplantam controles de funcionalidade, de eficiência ede manutenção; cuida das condições de operacionalidade do prédio; faz proje ções; sugere providências; analisa as áreas críticas; orienta as M anutenções Preventivas e Corretivas no sentido de possibilitar a segurança máxima da continuidade operacional, prevenindo, dessa forma, acidentes, falhas, interrupçoes, omissões, descontinuidades e emergências. As- 
sim, prevê necessi dades atuais e futuras; planeja carências; implanta programas e projetos; aloca verbas; providencia recursos humanos, materiais, capacitações, de acordo com cronogramas físico-financeiros junto à Administração do EAS 4 . A gestão de edifícios éfeita por meio do gerenciamento de facilidades, que éfundamental para a sustentabilidade nos edifícios, mediante o consumo racional de energia, água e materiais, diminuindo o volume de resíduos gerados, possibilitando a garantia das funções dos subsistemas por toda a vida útil do edifício. A gestão da informação contribui para o desenvolvimento dos programas estabelecidos pelos gestores ${ }^{13}$. Com relação aos quesitos de segurança na gestão e operação dos sistemas de água, energia elé trica, gases, vapores, efluentes líquidos, proteção contra descarga elétrica, climatização, combatea incêndio, alerta eemergência, tel efonia e rede lógica, $73,8 \%$ dos EAS os atendem parcialmente, pois apenas $26,2 \%$ gerenciam todos os itens. Segundo Karman et al. ${ }^{4}$, o EAS é um "permanente cantei ro de obras" e uma "instituição à espera de conclusão". Cada vez maiores são as necessidades de adequações e alterações, em função de avanços tecnológicos, e de reformas; obras novas se sucedem; equipamentos e instalações são substituídos com frequência; a vidaútil dos produtos e o desafio contra a obsolescência física e funcional geram a necessidade de planejamento, de ação e de investimento cada vez mais ágeis.

- Segundo Karman et al.,14, os responsáveis pela manutenção dos EAS devem possuir conhecimento técnico adequado para o planejamento e a execução das atividades operacionais e de manutenção predial, gerando rotinas detrabalho que abranjam instalações e equipamentos do Edifício. Devem criar arquivos, informatizar os processos, capacitar as equipes com o objetivo deatender a comunidade no quesito da saúde. Todos os EAS pesquisados possuem responsáveis pelas equipes de manutenção predial, mas a maioria não possui qualificação específica, sendo necessária, portanto, a sua capacitação para a gestão adequada dos serviços. Existe pouca valorização dessa atividade em relação às diretrizes do corpo administrativo das unidades, assim como pouca destinação de recursos financeiros para possibilitar as ações e os serviços de manutenção predial nos edifícios, especialmente as "administrativas, preventivas e de rotina". Ainda prevalecem as manutenções corretivas, que, segundo Karman et al. ${ }^{4,14}$, ocorrem por ausência ou deficiência nas programações das demais manutenções, gerando maiores custos funcionais efinanceiros. Cons- tatou-se que, além das equipes próprias para a manutenção predial, em 91,8\% dos EAS contratam-se serviços terceirizados (Tabela 2), para os quais também são demandados profissionais especializados, com conhecimento técnico para a gestão e o controle dos processos nas diversas etapas de contratação até à conferência dos mesmos, tanto nos prédios públicos quanto nos privados. Para assegurar a manutenção e a operacionalidade, é importante existir equipes com profissionais adequados. N a terceirização e na quarteirização de serviços, todos os envolvidos compartilham a responsabilidade pela qualidade $e^{15}$.

A Tabela 4 mostra o cruzamento entre o tipo de EAS e as variáveis necessárias para a obtenção dos alvarás de funcionamento e sanitário exigidos para as atividades assistenciais desaúde. Existe correlação entre os dados, pois, para a liberação do alvará sanitário, é imprescindível que o projeto arquitetônico esteja aprovado na VISA, assim como, para a emissão de alvará de funcionamento, énecessário que o projeto arquitetônico esteja aprovado na Prefeitura de acordo com a regulação urbana. As U BS e U PA pesquisadas não possuem tais alvarás, ao contrário das unidades hospitalares e outros como Farmácia, Banco de Leite, cujos programas de governo exigem médicose farmacêuticos como responsáveistécnicos. Inclusive, no item de monitoramento da qualidade da água, essas unidades especializadas possuem a exigência desse controle, que éfeito com a garantia deselos de qualidade dos Laboratórios. A RDC $63 / 2011^{8}$ confirma que os serviços públicos não precisam ter o Alvará Sanitário, mas devem ser visitados pela VISA local e seguir todos os requisitos exigidos para as BPF, que são os componentes da Garantia da Qualidade. Entretanto, para a ONA, o alvará sanitário é exigência e condição para se habilitar ao processo de acreditação.

A Tabela 5 mostra o cruzamento entre o tipo de EAS e as variáveis pesquisadas quanto aos aspectos físicos, como projetos aprovados pelo Corpo de Bombeiros e pela concessionária de energia elétrica. Identifica também o cruzamento com aspectos de manutenção. Outros pré- requisitos avaliados foram os cruzamentos entre os PPRA e os PGRSS eos diversos tipos deEAS pesquisados.

A Tabela 6 apresenta o cruzamento entre 0 tipo de EAS e as variáveis pesquisadas referentes aos quesitos de segurança aos usuários adotados para a verificação da qualidade no atendimento assistencial. A maior parte dos itens indica haver associação significativa no cruzamento entre os EAS e as variáveis descritas na tabela. 


\begin{tabular}{|c|c|c|c|c|c|}
\hline Item pesquisado/Unidade & $\underset{(n / \%)}{\operatorname{Sim}}$ & $\begin{array}{l}\text { Não } \\
(n / \%)\end{array}$ & $\begin{array}{l}\text { Parcial } \\
(\mathrm{n} / \%)\end{array}$ & $\mathrm{P} *$ & $\begin{array}{l}\text { Não se aplica ou } \\
\text { não informado }\end{array}$ \\
\hline \multicolumn{6}{|l|}{ Possui Alvará de Funcionamento? } \\
\hline UBS & 2 & 32 & 0 & & 0 \\
\hline Unidades especializadas & 4 & 8 & 0 & & 1 \\
\hline Unidades hospitalares & 3 & 0 & 0 & 0,000 & 2 \\
\hline Unidades saúde mental & 0 & 5 & 0 & & 0 \\
\hline UPA & 0 & 4 & 0 & & 0 \\
\hline \multicolumn{6}{|l|}{ Possui Alvará Sanitário? } \\
\hline UBS & 2 & 32 & 0 & & 0 \\
\hline Unidades especializadas & 3 & 6 & 2 & & 2 \\
\hline Unidades hospitalares & 4 & 1 & 0 & 0,001 & 0 \\
\hline Unidades saúde mental & 0 & 5 & 0 & & 0 \\
\hline UPA & 0 & 4 & 0 & & 0 \\
\hline \multicolumn{6}{|c|}{ Possui projeto arquitetônico aprovado na Vigilância Sanitária? } \\
\hline UBS & 2 & 22 & 0 & & 10 \\
\hline Unidades especializadas & 8 & 3 & 0 & & 2 \\
\hline Unidades hospitalares & 3 & 1 & 1 & 0,000 & 0 \\
\hline Unidades saúde mental & 0 & 4 & 0 & & 1 \\
\hline UPA & 0 & 1 & 0 & & 3 \\
\hline
\end{tabular}

*Valor de corte de 0,05: valores menores indicam que há uma associação significativa Fonte: Autora.

A Tabela 7 apresenta o cruzamento entre 0 tipo de EAS eas variáveis referentes às condições básicas de acessibilidade para pessoas com ne cessidades especiais: sinal ização de fácil entendimento e visualização que oriente o acesso e o fluxo, com a indicação de saídas de emergência no prédio. Outras variáveis pesquisadas foram 0 monitoramento da limpeza e da desinfecção dos reservatórios de água, além da análise e do controle da potabilidade da água periodicamente. Esses itens indicam haver associação significativa do cruzamento entre os EAS e essas variáveis.

A Tabela 8 descreve o cruzamento entre o tipo de EAS e as variáveis da gestão dos sistemas de água, energia elétrica, gases, vapores, efluentes líquidos, proteção contra descarga elétrica, climatização, combate a incêndio, al erta e emergência, telefonia, rede de lógica, assim como de equipamentos de infraestrutura de acordo com o porte e o dimensionado às necessidades do serviço.

Quanto à sistemática preventiva e corretiva das instalações e dos equipamentos, outra variável estudada, constatou-se que é feita a manutenção corretiva na maior parte dos EAS, não existindo ainda a programação das preventivas. A administração do EAS deve compreender o sistema de manutenção em relação à efetividade operacional quando o prédio é novo e ao longo da sua existência, de modo que não sejam necessárias paralisações para reformas e substituições, situações que comprometem o funcionamento adequado aos usuários, principalmente o estabelecimento público, que está programado para uma determinada população deabrangência ${ }^{4}$. Foi constatado, em $24,6 \%$ dos prédios, o planejamento das obras e que a maioria $(73,8 \%)$ cumpre parcialmente tal quesito. Com relação aos "sistemas de conservação e recuperação da estrutura física predial e do mobiliário, de acordo com o porte e o dimensionado às necessidades do serviço", $68,9 \%$ atendem ao quesito, somente 6,6\% não atende e 24,6\% parcialmente. Percebesepreocupação com o estado de conservação dos edifícios e dos mobiliários dos EAS estudados. Quase não são feitos "Planos de Contingências" na gestão da estrutura físico-funcional. 0 "Plano de Contingência ou Planejamento de Riscos" tem o objetivo de descrever as medidas a serem tomadas pelos gestores, incluindo a ativação de processosmanuais, para fazer com que seus processos vitais voltem a funcionar plenamente. No caso dos Estabelecimentos de Saúde, devem ser previstas ações e procedimentos para eventuais problemas que possam ocorrer e inviabilizar 0 atendimento proposto aos usuários. Em alguns estabelecimentos foi constatada a existência de 
Tabela 5. Aspectos físicos e de manutenção dos estabelecimentos. M inas Gerais, 2011.

\begin{tabular}{|c|c|c|c|c|c|}
\hline Item pesquisado/Unidade & $\underset{(n / \%)}{\operatorname{Sim}}$ & $\begin{array}{l}\text { Não } \\
(n / \%)\end{array}$ & $\begin{array}{c}\text { Parcial } \\
(\mathrm{n} / \%)\end{array}$ & $p^{*}$ & $\begin{array}{l}\text { Não se aplica ou } \\
\text { não informado }\end{array}$ \\
\hline \multicolumn{6}{|c|}{ Possui projeto de Prevenção de Combate a Incêndio e Pânico aprovado junto ao Corpo de Bombeiro? } \\
\hline UBS & 2 & 25 & 1 & & 6 \\
\hline Unidades especializadas & 8 & 2 & 2 & & 1 \\
\hline U nidades hospitalares & 3 & 0 & 2 & 0,000 & 0 \\
\hline Unidades saúde mental & 0 & 4 & 1 & & 0 \\
\hline UPA & 0 & 0 & 0 & & 4 \\
\hline \multicolumn{6}{|l|}{ Projeto aprovado na CEMIG? } \\
\hline UBS & 0 & 34 & 0 & & 0 \\
\hline U nidades especializadas & 0 & 13 & 0 & não & 0 \\
\hline U nidades hospitalares & 0 & 5 & 0 & há & 0 \\
\hline Unidades saúde mental & 0 & 5 & 0 & teste & 0 \\
\hline UPA & 0 & 4 & 0 & & 0 \\
\hline \multicolumn{6}{|l|}{ Responsável pela equipe de manutenção predial? } \\
\hline UBS & 0 & 34 & 0 & & 0 \\
\hline U nidades especializadas & 0 & 13 & 0 & não & 0 \\
\hline Unidades hospitalares & 0 & 5 & 0 & há & 0 \\
\hline Unidades saúde mental & 0 & 5 & 0 & teste & 0 \\
\hline UPA & 0 & 4 & 0 & & 0 \\
\hline \multicolumn{6}{|c|}{ Equipe de manutenção é a mesma de equipamentos? } \\
\hline UBS & 34 & 0 & 0 & & 0 \\
\hline U nidades especializadas & 13 & 0 & 0 & & 0 \\
\hline U nidades hospitalares & 1 & 4 & 0 & 0,000 & 0 \\
\hline Unidades saúde mental & 5 & 0 & 0 & & 0 \\
\hline UPA & 4 & 0 & 0 & & 0 \\
\hline \multicolumn{6}{|l|}{ PPRA atualizado e implantado? } \\
\hline UBS & 1 & 32 & 1 & & 0 \\
\hline Unidades especializadas & 1 & 12 & 0 & & 0 \\
\hline Unidades hospitalares & 4 & 0 & 1 & 0,000 & 0 \\
\hline Unidades saúde mental & 0 & 5 & 0 & & 0 \\
\hline UPA & 0 & 4 & 0 & & 0 \\
\hline \multicolumn{6}{|l|}{ PGRSS implantado? } \\
\hline UBS & 1 & 19 & 14 & & 0 \\
\hline U nidades especializadas & 2 & 7 & 2 & & 2 \\
\hline Unidades hospitalares & 3 & 0 & 2 & 0,005 & 0 \\
\hline Unidades saúde mental & 2 & 3 & 0 & & 0 \\
\hline UPA & 0 & 3 & 1 & & 0 \\
\hline
\end{tabular}

*Valor de corte de 0,05: valores menores indicam que há uma associação significativa Fonte: Autora.

"Plano de Contingência" para a substituição de algum equipamento que deixe defuncionar, contando com a colaboração de outras Unidades das respectivas redes, como no caso de Betim e na FHEM IG. Existem paralisações passíveis de serem programadas por meio de estudos de custos/benefícios para subsidiar as "tomadas de decisão" da "alta direção"; já a interrupção não programada no fornecimento de oxigênio ou energia elétrica (mesmo o gerador pode fal har na falta de energia, na ausência de testes rotineiros de monitoramento de seu funcionamento), por exemplo, no caso de pacientes críticos, tem preço em vidas e sequelas, ocorrências inadmissíveis na concepção da manutenção programada e na continuidade operacional ${ }^{4}$.

A qualidade é um atributo tão importante nos serviços de saúde, que diversas legislações têm sido implementadas para a regulação e o controle dos vários processos nos estabelecimentos, como a RDC 63/20118, que dispõe sobre os "Requisitos de Boas Práticas de Funcionamento para os Serviços deSaúde" edefineos serviços de saúde como estabelecimentos destinados a pres- 
Tabela 6. Tipo de estabelecimento e gestão da estrutura físico-funcional. Minas Gerais, 2011.

\begin{tabular}{|c|c|c|c|c|c|}
\hline Item pesquisado/Unidade & $\begin{array}{l}\operatorname{Sim} \\
(\mathrm{n} / \%)\end{array}$ & $\begin{array}{l}\text { Não } \\
(\mathrm{n} / \%)\end{array}$ & $\begin{array}{l}\text { Parcial } \\
(\mathrm{n} / \%)\end{array}$ & $\mathrm{P}^{*}$ & $\begin{array}{l}\text { Não se aplica ou } \\
\text { não informado }\end{array}$ \\
\hline \multicolumn{6}{|c|}{ Profissionais com capacitação compatível, dimensionadas às necessidades do serviço } \\
\hline UBS & 0 & 0 & 34 & & 0 \\
\hline Unidades especializadas & 0 & 0 & 13 & & 0 \\
\hline Unidades hospitalares & 2 & 2 & 1 & 0,000 & 0 \\
\hline Unidades saúde mental & 0 & 0 & 5 & & 0 \\
\hline UPA & 0 & 0 & 4 & & 0 \\
\hline \multicolumn{6}{|c|}{$\begin{array}{l}\text { Condições operacionais e de infraestrutura para execução das atividades, considerando o perfil e a } \\
\text { complexidade da organização }\end{array}$} \\
\hline UBS & 0 & 0 & 34 & & 0 \\
\hline Unidades especializadas & 0 & 0 & 13 & & 0 \\
\hline Unidades hospitalares & 2 & 0 & 3 & 0,012 & 0 \\
\hline Unidades saúde mental & 0 & 0 & 5 & & 0 \\
\hline UPA & 0 & 0 & 4 & & 0 \\
\hline \multicolumn{6}{|l|}{ Planejamento de obras } \\
\hline UBS & 5 & 1 & 28 & & 0 \\
\hline Unidades especializadas & 8 & 0 & 5 & & 0 \\
\hline Unidades hospitalares & 2 & 0 & 3 & 0,016 & 0 \\
\hline Unidades saúde mental & 0 & 0 & 5 & & 0 \\
\hline UPA & 0 & 0 & 4 & & 0 \\
\hline \multicolumn{6}{|c|}{ Projetos atualizados e em conformidade com o edificado } \\
\hline UBS & 5 & 21 & 1 & & 7 \\
\hline Unidades especializadas & 8 & 2 & 3 & & 0 \\
\hline Unidades hospitalares & 2 & 1 & 2 & 0,000 & 0 \\
\hline Unidades saúde mental & 0 & 3 & 0 & & 2 \\
\hline UPA & 1 & 0 & 0 & & 3 \\
\hline
\end{tabular}

*Valor de corte de 0,05: valores menores indicam que há uma associação significativa Fonte: Autora.

tar assistência à população na prevenção de doenças, no tratamento, na recuperação e na reabilitação. 0 serviço desaúdedeve desenvolver ações no sentido de estabelecer uma política de qualidade envolvendo estrutura, processo e resultado na sua gestão dos serviços, assim como utilizar a Garantia da Qualidade como ferramenta de gerenciamento. Os quesitos exigidos na infraestrutura para as "Boas Práticas" são basicamente os mesmosprevistos no nível 1 do M anual da ONA ${ }^{6}$.

No estudo efetuado nos 61 EAS, constata-se ser muito recente a mudança de paradigmas na gestão da manutenção predial dos edifícios. Os gerentes se queixam de falta de intervenção da direção nos serviços demanutenção. Portanto, éimprescindível identificar as principais diferençasentre os pontos de vista dos dirigentes, que devem estabelecer ações estratégicas, edo pessoal responsável pelas atividades operacionais, para que as informações e os conhecimentos técnicos, nas atividadesinterdisciplinares, possibilitem a eficiência nos processos de manutenção do edifício ${ }^{15}$.
Com a mudança dos paradigmas organizacionais e estruturais dos EAS em função das políticas e diretrizes assistenciais, tornaram-se necessárias as atividades dos profissionais especialistas em arquitetura e engenharia para construções, reformas, ampliações e adequações físicas de acordo com os modelos contemporâneos, assim como tecnologias disponíveis com toda a complexidade das instalações prediais. 0 perfil do gestor de serviços de manutenção predial evoluiu de artífice habilidoso para pessoa detentora de nível universitário, de cursos de pós-graduação e aperfeiçoamentos. Cabe a esse gestor 0 domínio da complexidade e da tecnicidade do edifício, estar atento às demandas de reparos, conservação, operacionalidade, avaliação decustos/benefícios, orçamentos, novos investimentos, equipamentos, obras e instalações, para subsidiar a adequada tomada de decisão, demandas estas muitas vezes oriundas da "alta direção" do hospital, pessoas com desconhecimento técnico dos assuntos envolvidos $\mathrm{s}^{4,16}$. 
Tabela 7. Tipo de estabelecimento e gestão da estrutura físico-funcional. M inas Gerais, 2011.

\begin{tabular}{|c|c|c|c|c|c|}
\hline Item pesquisado/Unidade & $\begin{array}{l}\operatorname{Sim} \\
(\mathrm{n} / \%)\end{array}$ & $\begin{array}{l}\text { Não } \\
(\mathrm{n} / \%)\end{array}$ & $\begin{array}{l}\text { Parcial } \\
(\mathrm{n} / \%)\end{array}$ & $\mathrm{P}^{*}$ & $\begin{array}{l}\text { Não se aplica ou } \\
\text { não informado }\end{array}$ \\
\hline \multicolumn{6}{|c|}{ Condições de acessibilidade para pessoas com necessidades especiais } \\
\hline UBS & 8 & 1 & 25 & & 0 \\
\hline Unidades especializadas & 8 & 0 & 5 & & 0 \\
\hline Unidades hospitalares & 4 & 1 & 0 & 0,003 & 0 \\
\hline U nidades saúde mental & 0 & 0 & 5 & & 0 \\
\hline UPA & 2 & 0 & 2 & & 0 \\
\hline \multicolumn{6}{|c|}{$\begin{array}{l}\text { Sinalização de fácil entendimento e visualização que oriente o acesso a todas as áreas da organização, } \\
\text { com controle nas áreas restritas }\end{array}$} \\
\hline UBS & 6 & 12 & 16 & & 0 \\
\hline Unidades especializadas & 11 & 1 & 1 & & 0 \\
\hline Unidades hospitalares & 3 & 1 & 1 & 0,000 & 0 \\
\hline U nidades saúde mental & 0 & 1 & 4 & & 0 \\
\hline UPA & 2 & 0 & 2 & & 0 \\
\hline \multicolumn{6}{|c|}{ Saídas de emergência claramente sinalizadas e de fácil compreensão } \\
\hline UBS & 6 & 28 & 0 & & 0 \\
\hline Unidades especializadas & 10 & 3 & 0 & & 0 \\
\hline Unidades hospitalares & 3 & 1 & 1 & 0,000 & 0 \\
\hline U nidades saúde mental & 0 & 5 & 0 & & 0 \\
\hline UPA & 2 & 2 & 0 & & 0 \\
\hline \multicolumn{6}{|c|}{ M onitora a análise e controle da potabilidade da água periodicamente } \\
\hline UBS & 2 & 32 & & & 0 \\
\hline U nidades especializadas & 3 & 7 & & & 3 \\
\hline Unidades hospitalares & 5 & 0 & & 0,000 & 0 \\
\hline U nidades saúde mental & 0 & 5 & & & 0 \\
\hline UPA & 0 & 4 & & & 0 \\
\hline
\end{tabular}

*Valor de corte de 0,05 : valores menores indicam que há uma associação significativa

Fonte: Autora.

Tabela 8. Tipo de estabelecimento e gestão da estrutura físico-funcional. M inas Gerais, 2011.

$\begin{array}{lccccc}\text { I tem pesquisado/Unidade } & \begin{array}{c}\text { Sim } \\ (\mathrm{n} / \%)\end{array} & \begin{array}{c}\text { Não } \\ (\mathrm{n} / \%)\end{array} & \begin{array}{c}\text { Parcial } \\ (\mathrm{n} / \%)\end{array} & \mathrm{P}^{*} & \begin{array}{c}\text { Não se aplica ou } \\ \text { não informado }\end{array}\end{array}$

Gerencia, opera e controla os sistemas de água, energia elétrica, gases, vapor, efluentes líquidos, proteção contra descarga elétrica, climatização, combate a incêndio, alerta e emergência, telefonia e rede lógica

$\begin{array}{lrrrrr}\text { U BS } & 0 & 4 & 30 & & 0 \\ \text { Unidades especializadas } & 0 & 9 & 4 & & 0 \\ \text { Unidades hospitalares } & 0 & 3 & 2 & 0,000 & 0 \\ \text { Unidades saúde mental } & 0 & 0 & 5 & & 0 \\ \text { UPA } & 0 & 0 & 4 & & 0\end{array}$

Gerencia, opera e controla os equi pamentos de infraestrutura (elevadores, caldeiras, geradores de energia, monta-cargas, entre outros), de acordo com o porte e o dimensionamento às necessidades do serviço

UBS

Unidades especializadas

Unidades hospitalares

Unidades saúde mental

UPA

Planos de contingências

UBS

U nidades especializadas

U nidades hospitalares

U nidades saúde mental

UPA

*Valor de corte de 0,05: valores menores indicam que há uma associação significativa Fonte: Autora. 
Todos os 61 EAS pesquisados possuem responsável pela equipe de manutenção predial. Alguns sem especialização adequada, mas tendo que se capacitarem com a prática e a vivência profissional, sobrecarregados com diversas outras atividades.

Outra questão a ser observada é a hotelaria hospitalar, queéa gestão em hospitalidadee humanização, na qual há necessidade de mudança e transformação dos hospitais nas estruturas físicas, com mudanças de paradigmas, pois os edifícios possuíam internações que antes aprisionavam os pacientes, mas a tendência atual éque sejam transformados em "prédios inteligentes", com processos internos focados no atendimento de qualidade. Os indivíduos que compõem as equipes do hospital devem estar focados na cura dos pacientes, assim como no bem-estar geral dos clientes envolvidos ${ }^{17}$. Observam-se as mudanças e as transformações necessárias em basicamente todos os hospitais da FHEMIG, cujas estruturas físicas, na maioria das vezes, são da década de 30, alguns com funções completamente distintas da assistência atual.

Outra mudança de paradigma são os modelos construtivos dos edifícios destinados às assistências da atenção primária e às especialidades. Conforme constatado na pesquisa, das 34 U BS de Betim, cerca da metade funciona em sede própria; as demais estão instaladas em construções, na maioria, de uso residencial, alugadas, carecendo de adequações físicas para o funcionamento mínimo das atividades necessárias. Percebe-se, portanto, que essa situação implica em estabelecimentos, muitas vezes sem projetos arquitetônicos passíveis de aprovação junto à regulação urbana do município, assim como das VISA municipais e/ou estaduais; por conseguinte, não possuidores de alvarás de funcionamento e sanitário. Até poucos anos atrás não existiam financiamentos para construção, reforma e manutenção de áreas físicas das UBS.

Recentemente, os governos federal e estadual estão disponibilizando recursos financeiros para esse fim mediante programas da SES destinados à construção de novas sedes. Em 2011 foi lançado um programa do Ministério da Saúde para "requalificação", ampliação, de modo a possibilitar reformas e recuperação de áreas físicas para as U BS. Tais recursos, ainda insuficientes, precisam de complementação de recursos próprios dos municípios, que, na grande maioria, já disponibilizam recursos elevados para o custeio das Unidades, entre diversas outras prioridades. Há, portanto, com a priorização dos financiamen- tos de recursos públicos, uma tendência em aprimorar as construções das novas unidades de saúde mediante alternativas de modelos construtivos adequados aos serviços de atenção primária da saúde, assim como de atendimentos especializados e de saúde mental.

A ONA trabalha com o conceito de evento sentinela (Norma Orientadora 4), que é a ocorrência inesperada ou a variação do processo envolvendo óbito, qualquer lesão física ou psicológica, ou riscos dos mesmos. Tais eventos podem ocorrer com o profissional da saúde, o cliente ou o paciente. Assim, ocorrências que envolvam a infraestrutura e os usuários de uma instituição de saúde podem comprometer a condição de "acreditado" 6 . As condições que causam erro humano podem ser minimizadas ou eliminadas no contexto hospital ar, levando à diminuiç̧ão deeventos adversos e de mortes evitáveis, contribuindo para a segurança e a qualidade na assistência do paciente. Esse resultado é possível na medida em que o "Projeto de Hospitais Sentinela" está apresentando os resultados das informações qualificadas sobre o desempenho dos produtos de saúde utilizados no Brasil e notificando os eventos adversos e as queixas técnicas de produtos de saúde, sangue e hemoderivados, materiais e equipamentos médico-hospitalares ${ }^{18}$.

\section{Conclusão}

Deacordo com as "Boas Práticas de Qualidade" e os itens de verificação da "gestão físico-funcional" da ONA, o serviço de saúde deve prover infraestrutura física, recursos humanos, equipamentos, insumos e materiais necessários à operacionalização do serviço, de acordo com a demanda, a modalidade de assistência prestada e a legislação vigente.

Com relação à gestão deinfraestrutura, o serviço de saúde deve ter projeto básico de arquitetura atualizado, em conformidade com as atividades desenvolvidas e aprovado pela VISA e demais órgãos competentes; suas instalações prediais deágua, esgoto, energia elétrica, gás, climatização, proteção e combate a incêndio, comunicação e outras existentes devem atender às exigências dos códigos de obras e posturas locais, assim como normas técnicas pertinentes; já as instal ações físicas dos ambientes externos einternos devem ser mantidas em boas condições de conservação, segurança, organização, conforto e limpeza. Ações de gerenciamento dos riscos de acidentes inerentes às suas atividades devem ser 
desenvolvidas; seus edifícios devem dotados de iluminação e ventilação compatíveis com as atividades; neles deve-se garantir a qualidade da água necessária ao funcionamento, por meio da limpeza dos reservatórios de água a cada seis meses, e ser mantido o registro da capacidade e da limpeza periódica dos reservatórios de água, assim como garantir a continuidade do seu fornecimento, mesmo em caso de interrupção do fornecimento pela concessionária, principalmente nos locais em que a água é considerada insumo crítico. Deveser garantida a continuidade do fornecimento de energia elétrica, em situações de interrupção pela concessionária, por meio de sistemas de energia elétrica de emergência, nos locais em que esta é considerada insumo crítico. As ações de manutenção preventiva e corretiva das instalações prediais devem ser garantidas de forma própria ou terceirizada.

Dessa forma, a O NA tem como objetivo contribuir para a melhoria contínua dos serviços de saúde tendo como foco a satisfação do cliente, considerando seus requisitos declarados ou não.

Pelo presente estudo, diante dos resultados obtidos nos 61 EAS pesquisados, constata-seque poucos estabelecimentos cumprem os quesitos de segurança imprescindíveis ao funcionamento mínimo, queéa garantia de infraestrutura física, de recursos humanos, de equipamentos, de insumos e materiais necessários à operacionalização do serviço de acordo com a demanda, a modalidade de assistência prestada e a legislação vigente, com qualidadee efetividade. Entretanto, com as mudanças de paradigmas nos modelos assistenciais nas RAS, os programas de qualidade vigentes, assim como a forma de financiamento dos sistemas de saúde irão colaborar para estabel ecimentos e serviços mais seguros, não só os pesquisados, mas para todos os implantados no País, públicos e privados. Os estabelecimentos estudados, provavelmente, refletem a situação nacional, que não deve ser muito diferente da situação constatada, qual seja, que os edifícios não são seguros com relação à gestão físicofuncional.

\section{Colaboradores}

GM Amorim participou ativamente na elaboração teórica, na execução, tabulação e discussão dos resultados. ECV Quintão participou ativamentena elaboração e execução do trabal ho. HM Junior participou na conceituação teórico-metodológica enas possibilidades de execução do trabalho. PRF Bonan orientou e participou ativamente em todas as etapas do trabalho, atuando na elaboração teórica, na execução, tabulação e discussão dos resultados.

\section{Agradecimentos}

À FHEM IG, FAPEMIG, Unimontes pela parceria para viabilizar o mestrado em Ciências da Saúde. Especialmente à FU NED, na pessoa de seu Presidente Augusto Monteiro Guimarães, pela recepção à atividade profissional nesta I nstituição desde abril de 2012. 


\section{Referências}

1. Elias P. Saúde no Brasil. São Paulo: Cortez-Cedec 1996.

2. Campos CEA. O desafio da integralidade segundo as perspectivas da vigilância da saúde e da saúde da família. Cien Saude Colet 2003; 8(2):569-584.

3. Mendes EV. As redes de atenção à saúde. Brasília: Organização Pan-Americana da Saúde; 2011.

4. Karman JB, Fiorentini D, Karman J N M. M anutenção hospitalar preditiva. São Paulo: Pini; 1994.

5. Reiling J. Safety by design. Safe design of healthcare facilities. Qual Saf Health Care 2006; 15(Supl. 1):i34-i40.

6. Organização Nacional de Acreditação (ONA). Manual brasileiro de acreditação hospitalar da Organização Nacional de Acreditação - ONA-3. Braśília; 2010

7. Det Norke Veritas (DNV). Curso de formação do auditor interno da Organização Nacional de Acre ditação (ONA). Brasília; 2010.

8. Agência Nacional de Vigilância Sanitária (ANVISA). Resolução da Diretoria Colegiada 63 de 25 de novembro de 2011. Dispõe sobre os requisitos de boas práticas de funcionamento para os serviços de saúde. Diário Oficial da União 2011; 28 nov.

9. Anvisa. Resolução da Diretoria Colegiada - RDC no 306, de 7 de dezembro de 2004. Dispõe sobre o Regulamento Técnico para o gerenciamento de re síduos de serviços de saúde. Diário Oficial da U nião 2004; 10 dez.

10. Conama. Resolução № 358,de 29 de abril de 2005. Dispõe sobre 0 tratamento e a disposição final dos resíduos dos serviços de saúde e dá outras providências. Diário Oficial da União 2005; maio 4.

11. Associação Brasileira de Normas Técnicas (ABNT). NBR 9050 Acessibilidade a edificações, mobiliário, espaços e equipamentos urbanos. Rio de Janeiro: ABNT; 2004.
12. Agência Nacional de Vigilância Sanitária (Anvisa) Resolução - RDC no 50, de 21 de fevereiro de 2002. Dispõe sobre o Regulamento Técnico para planejamento, programação, elaboração e avaliação de projetos físicos de estabelecimentos assistenciais de saúde.

13. Miquelin LC. Anatomia dos edifícios hospitalares. São Paulo: CEDAS; 1992.

14. Karman JB, Fiorentini D, Karman JNM. Manutenção incorporada à arquitetura hospitalar. Brasília: M S; 1995.

15. Csapski CA. Qualidade em estabelecimentos de saúde São Paulo: Editora SENAC; 1999. (Série Apontamentos).

16. Lee H, Scott D. Construindo relacionamentos para uma melhor manutenção. Jt Comm Saf Pacient J Qual 2008; 34(12):724-733.

17. Boeger M. Hotelaria hospitalar: gestão em hospitalidade e humanização. São Paulo: Editora Senac; 2009.

18. Agência Nacional de Vigilância Sanitária. Projeto H ospitais Sentinela [Internet]; 2001. [acessado 2012 nov 8]. Disponível em: http://www.anvisa.gov.br/ servicosaude/hsentinela/index.htm.

Artigo apresentado em 02/04/2012

Aprovado em 11/05/2012

Versão final apresentada em 15/05/2012 